NEUES AUS ITAS

\section{ITAS beim HGF-Strategiefonds erfolgreich}

ITAS ist an zwei Forschungsvorhaben beteiligt, die der Senat der Hermann von Helmholtz-Gemeinschaft Deutscher Forschungszentren als förderungswürdig angesehen hat und die zukünftig aus dem HGF-Strategiefonds finanziert werden. Es handelt sich um die Vorhaben "Global zukunftsfähige Entwicklung - Perspektiven für Deutschland", für das ITAS federführend ist, und das Vorhaben "Realisierung von CFK-Rumpfkomponenten unter Einbeziehung des Concurrent Engineering", bei der das Deutsche Zentrum für Luft- und Raumfahrt (DLR) die Federführung hat.

Der Strategiefonds wurde auf Beschluß der HGF-Mitgliederversammlung und des Senats der HGF eingerichtet. Ziel des Fonds ist es, durch enge Zusammenarbeit der Zentren mit der Wirtschaft innovative Projekte zu realisieren und Projekte der Grundlagenund Vorsorgeforschung mit strategischer Bedeutung durchzuführen. Die Vorhabensanträge an den Strategiefonds werden durch externe, international besetzte Gutachterkommissionen einer kritischen Prüfung unterzogen.

\section{HGF-Projekt "Global zukunftsfähige Entwicklung - Perspektiven für Deutschland"}

\section{von Reinhard Coenen, ITAS}

Generelles Ziel des Vorhabens ist es, die Umsetzung eines integrativen Konzepts einer nachhaltigen Entwicklung, das ökologische, ökonomische und soziale Belange gleichrangig berücksichtigt, durch Erarbeitung von Handlungsund Orientierungswissen zu unterstützen. Die Ziele des Vorhabens sind im einzelnen:

- $\quad$ Erarbeitung von Grundlagen und Kriterien für eine am Nachhaltigkeitskonzept orientierte zukünftige Prioritätensetzung für die Forschungs- und Technologiepolitik im allgemeinen und für die der HGF im besonderen;

- die Entwicklung von Handlungsoptionen zur Förderung einer nachhaltigen Entwicklung in verschiedenen gesellschaftlichen Aktivitäts- bzw. Bedürfnisfeldern und in ausgewählten Modellregionen;

- die Vernetzung bisher weitgehend isoliert durchgeführter Arbeiten zum Thema nachhaltige Entwicklung in den verschiedenen HGF-Einrichtungen, um Synergieeffekte zu nutzen.

Schließlich soll das Vorhaben einen Beitrag zur öffentlichen und wissenschaftlichen Debatte über Operationalisierung und Umsetzung des Konzepts einer nachhaltigen Entwicklung leisten. Im Rahmen des Vorhabens sollen insbesondere die Potentiale analysiert werden, die neue und verbesserte Technologien (Schlüsseltechnologien und aktivitätsfelderspezifische Technologien) zur Erreichung einer nachhaltigen Entwicklung bieten können.

Für den Ansatz des Vorhabens sind folgende konzeptionelle Elemente konstitutiv:

- Zentrale Ausgangsprämisse ist, daß die drei Dimensionen der Nachhaltigkeit gleichrangig und integriert behandelt werden müssen mit dem Ziel, Verbesserungen der ökonomischen und sozialen Lebensbedingungen mit der langfristigen Sicherung der natürlichen Lebensgrundlagen in Einklang zu bringen. Erst die Integration der drei Dimensionen überwindet die konzeptionelle Schwäche einer von wirtschaftlichen und sozialen Fragestellungen, Zielsetzungen und Rahmenbedingungen isolierten Ökologiediskussion und ermöglicht damit gerade auch einen strategischen Durchbruch für ökologische Belange (Abschlußbericht der Enquete-Kommission des 13. Deutschen Bundestages "Schutz des Menschen und der Umwelt": Konzept Nachhaltigkeit. Vom Leitbild zur Umsetzung. 1998, S. 87). Zugleich sind die politisch-institutionellen Rahmenbedingungen für eine Politik der Nachhaltigkeit als vierte Dimension eines integrativen Konzepts zu betrachten. Hierbei sind auch die internationalen Verflechtungen Deutschlands in der EU und in den weltwirtschaft- 
lichen und weltpolitischen Prozessen $\mathrm{zu}$ berücksichtigen.

- Weiter wird davon ausgegangen, daß der Strukturwandel, der Gesellschaft und Wirtschaft in Richtung auf eine ökologisch nachhaltige Entwicklung bewegen soll, eines Ansatzes bedarf, der Elemente der (Ressourcen-)Effizienz (d.h. Reduzierung des Stoff- und Energieverbrauchs je Produkt bzw. Dienstleistungseinheit), der Suffizienz (Einschränkung der Nutzung von Gütern/Dienstleistungen durch Veränderungen von Lebensstilen) und der Konsistenz (Erhöhung der Konsistenz anthropogener mit natürlichen Stoffströmen) verbindet. Gegenüber anderen vorliegenden Studien und auch aufgrund der spezifischen Kompetenzen der beteiligten HGFEinrichtungen sollen jedoch vor allem die Effizienz- und Konsistenzverbesserungspotentiale von Innovationen technologischer, aber auch sozioökonomischer und institutioneller Art unter Berücksichtigung der jeweiligen Realisierungsprobleme untersucht werden. Der integrative Ansatz erfordert aber ebenso, die Potentiale solcher Innovationen im Hinblick auf soziale und ökonomische Ziele der Nachhaltigkeit abzuschätzen und zu bewerten.

- Mit dem Leitbild einer nachhaltigen Entwicklung ist die Perspektive einer größeren Nachhaltigkeit bei der Befriedigung gesellschaftlicher und individueller Bedürfnisse gegenwärtiger und zukünftiger Generationen verbunden. Dies legt einen Ansatz nahe, der nicht an ökonomischen Sektoren anknüpft, sondern direkt von Bedürfnisbzw. Aktivitätsfeldern ausgeht. In Erweiterung etwa der Arbeiten der EnqueteKommission "Schutz des Menschen und der Umwelt" oder der Studie des Umweltbundesamtes "Nachhaltiges Deutschland" von 1997 wird sich das Strategiefondsvorhaben dabei nicht auf beispielhaft ausgewählte Handlungsfelder beschränken, sondern alle gesellschaftlich und wirtschaftlich relevanten Aktivitäten erfassen und in ihren gegenseitigen Wechselwirkungen berücksichtigen. Nur ein solch breiter Ansatz erlaubt es, geeignete Technologien, Strategien und Maßnahmen zu identifizieren und im Hinblick auf ihre Nachhaltigkeit be- werten zu können. Dieser Anspruch schließt jedoch nicht aus, daß die verschiedenen Aktivitätsfelder je nach ihrer Bedeutung für eine nachhaltige Entwicklung in ökologischer, ökonomischer und sozialer Hinsicht mit unterschiedlicher Analysetiefe bearbeitet werden. Im Strategiefondsvorhaben sollen die Aktivitätsfelder Mobilität, Bauen und Wohnen, Landwirtschaft und Ernährung, Information und Kommunikation sowie Freizeit und Tourismus detailliert behandelt werden, während andere Aktivitätsfelder wie z.B. Gesundheit oder Textilien und Bekleidung aus Kapazitätsgründen nur auf einem reduzierten Analyseniveau bearbeitet werden.

- Zur modellhaften Umsetzung des integrierten Konzeptes an konkreten Beispielen ist im Vorhaben ein regionaler bzw. ökosystemarer Zugang vorgesehen. Für bestimmte Küstengebiete in Norddeutschland und für intensiv landwirtschaftlich genutzte Regionen sollen Problemfelder, regionalisierte Nachhaltigkeitsziele und -indikatoren, Zielkonflikte, Nutzungskonzepte, Strategienotwendigkeiten und Forschungsund Technologiebedarf im Hinblick auf ein integratives Nachhaltigkeitskonzept analysiert werden.

Im einzelnen wird das Vorhaben folgende parallele und iterativ durchzuführende Arbeitsschritte umfassen.

- Die Erarbeitung von Vorschlägen für nationale Nachhaltigkeitsziele und Leitindikatoren in den verschiedenen Dimensionen, deren Diskussion mit gesellschaftlichen Gruppen und - soweit wie möglich deren Konkretisierung in entsprechenden Zielsystemen oder -vorgaben für die verschiedenen Aktivitätsfelder.

- Die Analyse von Defiziten ökologischer, ökonomischer und sozialer Art in diesen Aktivitätsfeldern im Hinblick auf eine nachhaltige Entwicklung mittels geeigneter Kriterien und Indikatoren auf der Basis von Istzustands- und Trendanalysen.

- Die Abschätzung von Effizienz- und Konsistenzpotentialen technologischer und auch sozioökonomischer und institutioneller Innovationen für eine nachhaltige Entwicklung in den jeweiligen Aktivitäts- 
feldern mittels Verfahren der Technikvorausschau und der Technikfolgenabschätzung sowie, ergänzend dazu, die Identifizierung und Analyse nicht-nachhaltiger Lebensstile und von Möglichkeiten und Folgen ihrer Veränderung. Besondere Bedeutung im Rahmen dieses Arbeitsschritts kommt dabei der Abschätzung der Potentiale von sektorenübergreifenden bzw. Schlüsseltechnologien zu, z.B. Informations- und Kommunikationstechniken, Biound Gentechniken, Neue Materialien, Energie- und Antriebstechniken, Mikround Nanotechniken.

- Die Identifikation geeigneter Maßnahmen und Instrumente zur Nutzung dieser Potentiale und die Ableitung entsprechender "Nachhaltigkeitsstrategien".

- Die Analyse der Wechselwirkungen zwischen den einzelnen Aktivitätsfeldern und der Möglichkeiten einer konsistenten Integration der aktivitätsfelderspezifischen Teilstrategien.

- Die Analyse der Realisierungs- und Umsetzungsbedingungen der Strategien, die quantitative und qualitative Abschätzung ihrer Folgen sowie auf dieser Basis die Entwicklung von entsprechenden Handlungsoptionen für Politik und Gesellschaft.

- Die Erarbeitung von Grundlagen und Kriterien für die Prioritätensetzung in der Forschungspolitik, insbesondere auch in der HGF, und die Identifikation von FuE-Bedarf, der im Hinblick auf eine nachhaltige Entwicklung von besonderer Bedeutung ist.

Der HGF-Senat ist bei seiner Entscheidung dem Votum des Gutachterausschusses gefolgt, vier der insgesamt zehn Arbeitspakete des Vorhabens zurückzustellen, d.h. die Analyse einiger Aktivitätsfelder und die regionalbezogenen Analysen mit der Empfehlung, diese in einem komplementären Antrag in die nächste Runde des Strategiefonds einzubringen, um das Vorhaben entsprechend zu erweitern.

\section{Beteiligte Einrichtungen}

- Forschungszentrum Karlsruhe (FZK)

- Institut für Technikfolgenabschätzung und Systemanalyse (ITAS)

(federführend)
- Forschungszentrum Jülich (FZJ)

- Programmgruppe Mensch, Umwelt, Technik (MUT)

- Programmgruppe Technikfolgenforschung (TFF)

- Institut für Sicherheitsforschung und Reaktortechnik (ISR)

- Deutsches Zentrum für Luft- und Raumfahrt (DLR)

- Hauptabteilung Verkehrsforschung (FF-VL)

- Abteilung für Systemanalyse und Technikbewertung (TT-STB) des Instituts für Technische Thermodynamik

- Datenfernerkennungszentrum

- GMD-Forschungszentrum Informationstechnik

- Institut für Systementwurfstechnik (SET)

- Forschungsinstitut für Rechnerarchitektur und Softwaretechnik (FIRST)

- Umweltforschungszentrum Leipzig (UFZ)

- Abteilung ökologische Ökonomie/ Umweltsoziologie (ÖKUS)

- Projektbereich Naturnahe Landschaften und ländliche Räume (NLLR)

- Alfred-Wegener-Institut für Polarund Meeresforschung (AWI)

- Sektion Ökologie und Ökophysiologie

- Projektbereich Küstenforschung und Küstenzonenmanagement.

\section{HGF-Projekt: Realisierung von CFK- Rumpfkomponenten unter Einbezie- hung des Concurrent Engineering (Kurztitel: "Schwarzer Rumpf")}

\section{von Matthias Achternbosch, ITAS}

Der Weltluftverkehr hat in den vergangenen Jahrzehnten große Zuwachsraten zu verzeichnen. Die prognostizierten Wachstumsraten lassen eine weitere Verdoppelung der Luftverkehrsleistungen in den nächsten 10 - 15 Jahren erwarten. Die Weiterentwicklung der technologischen Kompetenz im Flugzeugbau ist daher 
ein wirtschaftspolitisch zwingendes Zukunftsthema.

Faserverbundbauweisen verfügen über das Potential, den Druckrumpf (Größe etwa Airbus A 320) zukünftiger Verkehrsflugzeuge 5 bis 8 Tonnen leichter (ca. $30 \%$ ) zu bauen. Im Laufe eines Flugzeuglebens bedeutet dies eine Treibstoffersparnis von ca. 24 Millionen Litern Kerosin. Im harten Wettbewerb zur amerikanischen Flugzeugindustrie besteht zudem die Notwendigkeit, die Herstellkosten heutiger Metallrumpfbauweisen bis zum Jahr 2010 durch neue Faserverbundbauweisen um $40 \%$ zu reduzieren.

Im Rahmen eines HGF-Verbundprojektes, das unter der Leitung des Deutschen Zentrums für Luft- und Raumfahrt e. V. (DLR) von verschiedenen Instituten der DLR und des Instituts für Technikfolgenabschätzung und Systemanalyse (ITAS) des Forschungszentrums Karlsruhe durchgeführt wird, sollen die notwendigen Grundlagen für einen optimierten Entwicklungsprozeß für ausgewählte Rumpfkomponenten eines Verkehrsflugzeuges erarbeitet werden. Das DLR ist mit seinen im Cluster "Werkstoffe und Strukturen" zusammengeschlossenen Instituten (Institut für Strukturmechanik, Braunschweig, Institut für Bauweisenund Konstruktionsforschung, Stuttgart) und dem Institut für Antriebstechnik, Köln-Porz, an der Weiterentwicklung der entsprechenden Technologien national und auch international maßgeblich beteiligt. Hierzu werden sowohl der Entwurfsprozeß durch Nutzung der parallelen Vorgehensweise des Concurrent/Integrated Engineering (CIE) als auch die anzuwendenden Faserverbundbauweisen in optimierter Form gestaltet.

Die Verwendung von kohlenstoffaserverstärktem Kunststoff (CFK) statt der üblichen Metalllegierungen soll darüber hinaus den Wartungsaufwand erheblich verringern; Komfort und Crashsicherheit sollen verbessert werden und die Durchbrandsicherheit und die Lebensdauer erhöht werden. Eine derartige Verbesserung von Druckrümpfen ist nur mit einem Technologiesprung umsetzbar.

Das Institut für Technikfolgenabschätzung und Systemanalyse (ITAS) des Forschungszentrums Karlsruhe untersucht in diesem HGFProjekt die Umweltverträglichkeit bei der Herstellung, beim Einsatz und bei der Entsorgung von Rumpfkomponenten.
Ziel dieses Hauptarbeitspunktes ist die Bestimmung des mit der Herstellung, dem Einsatz und der Entsorgung der CFK-Rumpfkomponenten verbundenen Stoff- und Energieeinsatzes und der daraus resultierenden Emissionen mit Abluft, Abwasser und Abfall. Zusätzlich soll ein Vergleich mit Rumpfkomponenten aus herkömmlichen Aluminiumwerkstoffen durchgeführt werden. Hierfür müssen Stoff- und Energiebilanzen für CFK-Werkstoffe und konventionelle Werkstoffe für die einzelnen Phasen des Lebenszyklus von Rumpfkomponenten erstellt werden. Dies beinhaltet:

- die Gewinnung von Vorprodukten für die Herstellung unterschiedlicher CFK- und konventioneller Werkstoffe

- die Fertigung der Werkstoffe

- die Fertigung von Rumpfkomponenten

- die Gebrauchsphase von Flugzeugen (Start, Flug, Landungen, Wartung)

- Recycling bzw. Entsorgung von konventionellen bzw. CFK-Materialien für Flugzeugrümpfe.

$\mathrm{Zu}$ diesen einzelnen Schritten in den Lebenszyklen wird eine Gesamtsachbilanz durchgeführt, in der ein detailliertes Mengengerüst zu Stoffund Energieeinsatz, Emissionen, Abwässern und Abfällen aufgestellt wird. In einem nächsten Schritt erfolgt die Durchführung einer Wirkungsbilanz, in der die Auswirkungen der freigesetzten Stoffe auf Mensch und Umwelt abgeschätzt werden. Abschließend sollen die Ergebnisse zusammengefaßt und eine Bewertung der Einzelergebnisse anhand von Indikatoren bzw. Wirkungskategorien durchgeführt werden.

Das Projekt beginnt am 1. Juli 1999. Die Laufzeit beträgt 3 Jahre.

\section{Systemanalytischer Vergleich verschiedener Herstellungs- verfahren von Energieträgern aus biogenen Abfällen}

\author{
Dissertation am Institut für Technikfolgen- \\ abschätzung und Systemanalyse \\ von Andreas Arlt
}


Die energetische Nutzung von biogenen Abfällen als Energieträger wird aufgrund veränderter Rahmenbedingungen zunehmend dringend und erforderlich. Diese Tendenz wird sich voraussichtlich in den kommenden Jahren weiter fortsetzen. Aus diesem Grunde führt eine Arbeitsgruppe am Institut für Technikfolgenabschätzung und Systemanalyse (ITAS) des Forschungszentrums Karlsruhe für Technik und Umwelt zur Zeit ein Projekt zu diesem Themenbereich durch. Innerhalb dieses Projektes werden verschiedene Verfahren zur energetischen Nutzung von biogenen Abfälle hinsichtlich ihrer technischen, ökonomischen und umweltrelevanten Kennwerte systemanalytisch miteinander verglichen. Hierbei wird die komplette Verfahrenskette von der für die Hol-, Bring-, Transport- und Lagersysteme erforderlichen Logistik, über die für die Aufbereitung der Abfälle zum Energieträger eingesetzten Konditionierungsverfahren, bis hin zur nachfolgenden Verfahrenstechnik für die energetische Nutzung betrachtet.

In das übergeordnete Projekt am ITAS eingebettet ist die im März 1999 begonnene Dissertation, die sich mit dem systemanalytischen Vergleich der verschiedenen Verfahren zur Herstellung der Energieträger beschäftigt. Die ersten Ergebnisse des Projektes und der Dissertation werden als Schwerpunktthema in der 1. Ausgabe der TA-Datenbank-Nachrichten im Jahre 2000 vorgestellt werden.

Folgende Rahmenbedingungen und aktuellen Entwicklungen erfordern zunehmend eine energetische Nutzung biogener Abfälle:

- Aufgrund des in der BRD gesetzlich gegebenen Rahmens (Kreislaufwirtschafts- und Abfallgesetz, 1996; TA-Siedlungsabfall, 1993) ist die Deponierung von biogenen Abfällen über das Jahr 2005 hinaus gesetzlich nicht mehr gestattet. Alle Stoffe, die dann auf die Deponie verbracht werden, dürfen nur noch einen Glühverlust von kleiner 5\% aufweisen.

- Auf europäischer Ebene werden (bisher) keine gesetzlichen Anforderungen an die auf Deponien verbrachten Stoffe gestellt. Vielmehr wird in der in diesem Jahr verabschiedeten Europäischen Deponierichtlinie vorgeschrieben, daß bis zum Jahre 2004 die deponierte Menge der biologisch abbaubaren Stoffe auf 75 Gew.-\% und bis zum Jahre 2007 auf 50 Gew.-\% reduziert werden muß.

- Die Bundesrepublik Deutschland hat sich auf Grundlage des "Kioto-Protokolls" zur Klimarahmenkonvention verpflichtet, die Emissionen von Treibhausgasen $\left(\mathrm{CO}_{2}\right.$, $\mathrm{CH}_{4}, \mathrm{~N}_{2} \mathrm{O}, \mathrm{HFCs}, \mathrm{PFCs}, \mathrm{SF}_{6}$ ) im Zeitraum von 2008 bis 2012 um 21\% bezogen auf $1990 \mathrm{zu}$ reduzieren. Biogene Energieträger können hierbei aufgrund der (weitgehend) geschlossenen Kohlenstoffkreisläufe und dem damit verbundenen $\mathrm{CO}_{2}$-Entlastungspotential einen wesentlichen Beitrag leisten.

- Die Mitnutzung von schwach belasteten, biogenen Abfällen in Anlagen zur Wärmeund Stromerzeugung aus naturbelassenen Biobrennstoffen kann deren Wirtschaftlichkeit deutlich verbessern.

Für die einzelnen Arten an biogenen Abfällen (Klärschlamm, Hausmüll mit biogenen Anteilen, strukturarmer (holzarmer) Bioabfall, strukturreicher (holzreicher) Bioabfall, Schlämme der Zellstoff-, Papier- und Lebensmittelindustrie, Alt- und Industrieresthölzer) läßt sich zeigen, daß die bisher beschrittenen Entsorgungswege über das Jahr 2005 hinaus teilweise keine Problemlösung mehr darstellen.

\section{Gründe hierfür liegen in}

- der für eine hochwertige stoffliche Verwertung erforderlichen, jedoch in der Praxis nicht erreichten Homogenität der Abfälle (z.B. DSD-Fraktionen des Hausmülls),

- den hohen Kosten der separaten Erfassung, Sortierung und stofflichen Verwertung (z.B. DSD-Fraktionen des Hausmülls, Kompostierung von Bioabfällen),

- den zunehmenden Absatzproblemen des erzeugten Produkts aus den biogenen Abfällen bei gleichzeitig zunehmendem $\mathrm{Ab}$ fallaufkommen (z.B. Kompost),

- dem Verbot der Deponierung von nicht vorbehandeltem Abfall ab dem Jahre 2005 bei bisher hohem Deponierungsgrad (z.B. Klärschlamm, Industrieschlämme, Altholz) und

- der aufgrund toxischer Verunreinigungen erschwerten stofflichen Verwertung (z.B. 
Klärschlamm, Altholz, Bioabfall, Straßenbegleitgrün).

Aufgrund dieser Problemlage erscheint es sinnvoll, die biogenen Abfälle mit thermochemischen oder biologischen Verfahren energetisch $\mathrm{zu}$ verwerten. Als thermochemische Verfahren für eine nachfolgende energetische Nutzung kommen dabei die Verbrennung, die CoVerbrennung in Kohlekraftwerken, der Einsatz als Brennstoff in der Zement- und Stahlindustrie sowie die Vergasung mit verschiedenen Arten der Gasverwendung in Betracht. Darüber hinaus ist bei manchen Abfallarten die Kombination biologischer und thermochemischer Verfahren sinnvoll, wie eine aerobe Behandlung als erste Stufe und die thermochemische Behandlung als zweite Stufe oder eine anaerobe Behandlung mit Biogasgewinnung als erste Stufe und eine thermochemische Behandlung als zweite Stufe.

Für eine energetische Nutzung der biogenen Abfälle ist jedoch eine Aufbereitung unumgänglich, da diese in der Regel in einer Form vorliegen, in der sie für eine energetische Nutzung zunächst ungeeignet sind. Sie sind häufig feucht, haben einen geringen Heizwert, sind in ihrer Zusammensetzung und Stückigkeit inhomogen, bewirten pathogene Keime, verursachen Geruchsemissionen und sind mit toxischen Substanzen oder sonstigen Fehlwürfen verunreinigt. Für die biologischen und thermochemischen Verfahren zur energetischen Nutzung werden jedoch Substrate bzw. Brennstoffe benötigt, die weitgehend homogen, lagerfähig, möglichst schadstoffarm und hygienisiert sind. Für die Nutzung durch thermochemische Verfahren muss das biogene Material zusätzlich getrocknet werden. Wichtige physikalisch-chemische Anforderungen an das Substrat bzw. den Brennstoff für eine energetische Nutzung liegen demnach im Brennwert, dem Feuchtegehalt, der Stückigkeit und der Schadstoffbelastung.

Darüber hinaus werden vom Gesetzgeber für Anlagen zur energetischen Verwertung von Abfällen strenge Auflagen bezüglich der Schadstofffrachten und -konzentrationen im Abgas und Abwasser vorgegeben sowie Anforderungen an die Rückstände der thermochemischen Verfahren gestellt. Diese Umstände machen insbesondere ein umfassendes Qualitätsmanagement der in eine Aufbereitungsverfahrens- kette aufgegebenen, biogenen Abfälle sowie der darin produzierten Substrate bzw. Ersatzbrennstoffe erforderlich.

Im Rahmen der Dissertation werden die unterschiedlichen Konditionierungsverfahren für biogene Abfälle systemanalytisch verglichen und bewertet. Fragestellungen der Erfassungslogistik auf der Aufkommensseite sowie Fragestellungen der thermischen Verwertung, der hierfür eingesetzten Verfahren (Feuerungstechnik), der Verfahrensoptimierung für z. B. die Verringerung von Schadgasen usw. auf der Verwertungsseite sollen nicht Bestandteil der Systemanalyse sein. Als Schnittstellenparameter werden auf der Aufkommensseite die Aufkommensmenge, die chemisch-physikalischen Parameter und vor allem die problematischen Inhaltsstoffe des biogenen Abfalls bei der Anlieferung an einem (fiktiven) Zwischenlager (Abfalllager) dienen, welches als Bilanzraumgrenze auf der Aufkommensseite dient. Auf der Verwertungsseite sollen als Schnittstellenparameter die Anforderungen der verschiedenen thermochemischen bzw. biologischen Verwertungsmodule an den (Ersatz-)Brennstoff dienen. Gedacht ist auch auf dieser Seite des Bilanzraums an ein (fiktives) Zwischenlager (Brennstofflager), das als Bilanzraumgrenze fungiert und in dem die Substrate bzw. Ersatzbrennstoffe für eine nachfolgende (nicht betrachtete) thermische oder biologische Nutzung gelagert werden. 